\title{
Development of a Time-Dependent Friction Model for Frictional Aging at the Nanoscale
}

\author{
Seung Yub Baek ${ }^{1}$ and Kyungmok Kim² \\ ${ }^{1}$ Department of Mechanical Design, Induk University, 2 Wolgye-dong, Nowon-gu, Seoul 139-749, Republic of Korea \\ ${ }^{2}$ School of Aerospace and Mechanical Engineering, Korea Aerospace University, \\ 76 Hanggongdaehang-ro, Deogyang-gu, Goyang-si, Gyeonggi-do 412-791, Republic of Korea
}

Correspondence should be addressed to Kyungmok Kim; kkim@kau.ac.kr

Received 11 November 2015; Accepted 15 February 2016

Academic Editor: Serdal Kirmizialtin

Copyright (C) 2016 S. Y. Baek and K. Kim. This is an open access article distributed under the Creative Commons Attribution License, which permits unrestricted use, distribution, and reproduction in any medium, provided the original work is properly cited.

A model for describing frictional aging of silica is developed at the nanoscale. A cohesive zone is applied to the contact surface between self-mated silica materials. Strengthening of interfacial bonding during frictional aging is reproduced by increasing fracture energy of a cohesive zone. Fracture energy is expressed as a function of hold time between self-mated silica materials. Implicit finite element simulation is employed, and simulation results are compared with experimental ones found in the literature. Calculated friction evolutions with various hold times are found to be in good agreement with experimental ones. Dependence of mesh size and cohesive thickness is identified for obtaining accurate simulation result.

\section{Introduction}

Frictional aging is the contact phenomenon that interfacial bonding between two bodies is strengthened with increasing hold time. The strengthening of interfacial bonding is attributed to increase in the maximum frictional force. The maximum frictional force was found to increase logarithmically with hold time [1]. Frictional aging is widely observed in macroscale and nanoscale contacts [2-5]. Strength of the contact between rocks was found to increase with the age of the contact [2]. Rate-dependent friction behaviour of granular materials was studied [3]. It was observed that friction behaviour of granular materials was dependent upon humidity; the transition from velocity strengthening to velocity-weakening frictional behaviour was found under certain humid condition. The aging behaviour of a single nanoscale contact was investigated by conducting slide-holdslide experiments with silica [4]. In a slide-hold-slide friction experiment, one body was held for certain time after the initial slip between two bodies. Slip was then induced at contact, and both frictional force and relative displacement were measured. It was identified that measured frictional force increased with respect to hold time. Effect of oscillation on fiction in nanoscale contact was studied with atomic force microscopy (AFM) measurement [5]. Small-amplitude inplane oscillation of a tip in AFM was found to affect the kinetics of frictional aging. Despite some studies on frictional aging, little is found on simulation of frictional aging at the nanoscale due to lack of adequate description of interfacial bonding.

Cohesive zone law is a useful method for describing interfacial failure between two physical parts. Various cohesive zone models were developed and used for simulating damage such as creep, fatigue, fretting fatigue, and interfacial cracking at the macroscale and microscale [6-9]. Creeprupture of aluminium alloys was modelled with a timedependent bilinear cohesive zone law [6]. Fretting fatigue cracking was reproduced with a cohesive zone law using cycle-jump strategy [7]. Stress amplitude versus cycles-tofailure curves were determined with aluminium alloys. At the microscale, interfacial cracking between alumina particles was simulated with a bilinear cohesive zone law [8]. Shear 


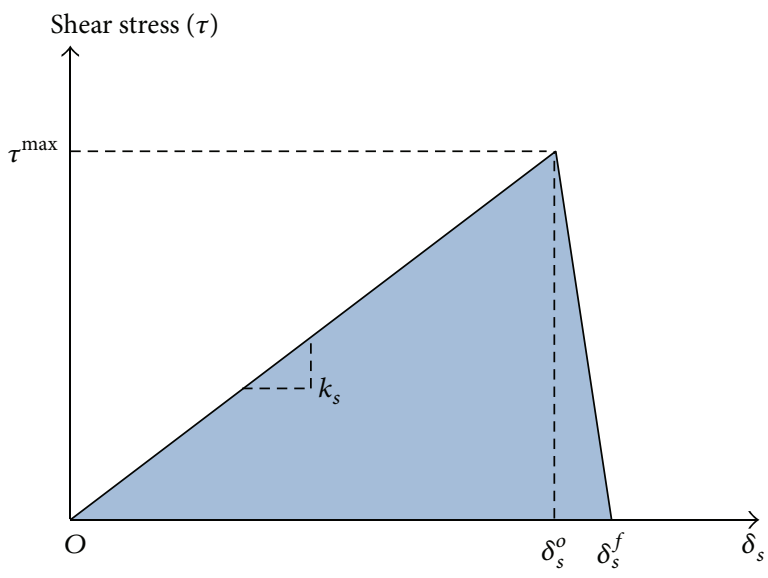

$\delta_{s}$ : shear displacement

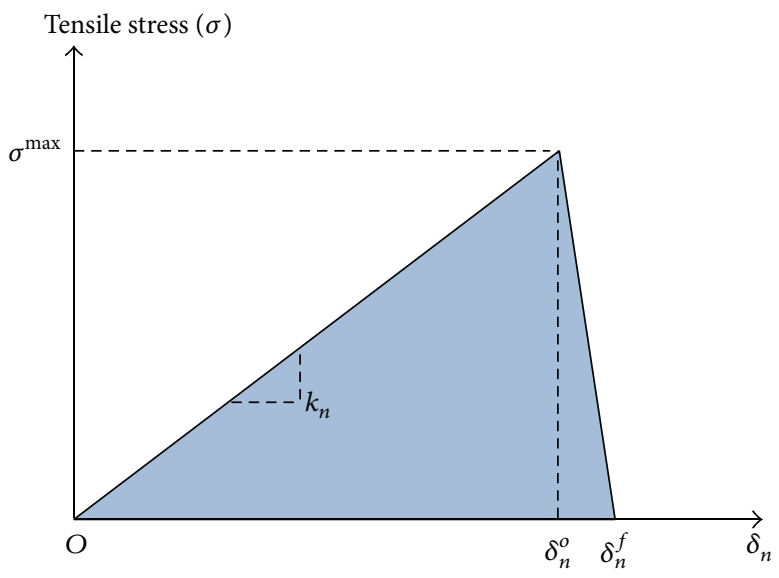

$\delta_{n}$ : normal displacement

FIgURE 1: Bilinear cohesive zone law.

and normal loads representing kinetic friction between a head and a cup in a hip prosthesis were induced to an alumina microstructure. Crack initiation and propagation were simulated with a two-dimensional finite element model. Softening behaviour of aluminium alloy under pure-tension loading was modelled with a nonlinear cohesive zone law [9]. Nonlinear softening behaviour of heat-treated and nonheat-treated specimens was described with the properties of a cohesive zone.

In this study, a bilinear cohesive zone law was proposed for describing static friction and frictional aging at the nanoscale. The strengthening of interfacial bonding resulting from frictional aging was achieved with the increase in cohesive energy. A three-dimensional finite element model was generated with a thin cohesive layer. The maximum frictional force and the relative displacement between two parts were determined after calculation. Direct comparison between simulation and experimental results was then employed.

\section{Time-Dependent Friction Model Using a Cohesive Zone Law}

When one body is pressed by the other, interfacial bonding is formed at the contact between two bodies. Once a tangential force is induced to the body, the bonding becomes weakened. If the tangential force is sufficient to break the bonding, slip initiates at the contact. Interfacial bonding is strengthened as hold time is increased (known as frictional aging). In other words, the maximum frictional force increases with increased hold time due to strengthening of interfacial bonding.

In this study, strengthening of interfacial bonding was described with the properties of a cohesive zone (i.e., cohesive energy). A bilinear cohesive zone law was used to describe interfacial bonding between two bodies. In a bilinear cohesive zone law, cohesive strength, cohesive stiffness, and displacement at failure determine fracture behaviour of a cohesive zone. In this study, cohesive strength was assumed as frictional strength.
Figure 1 shows the proposed bilinear cohesive zone law. Once the maximum stress of a cohesive element meets (1), partial slip at contact or separation is assumed to initiate:

$$
\left(\frac{\sigma}{\sigma^{\max }}\right)^{2}+\left(\frac{\tau}{\tau^{\max }}\right)^{2}=1,
$$

where $\sigma^{\max }$ is the maximum tensile stress associated with pull-off adhesion strength and $\tau^{\max }$ is the maximum shear stress related to static friction.

If a displacement continues to increase after satisfying (1), stress is then reduced according to a softening curve. A softening curve is described with the damage variable $(D)$ defined as

$$
D=\frac{\delta^{f}\left(\delta^{\max }-\delta^{o}\right)}{\delta^{\max }\left(\delta^{f}-\delta^{o}\right)},
$$

where $\delta^{\max }$ is total displacement determined with the normal displacement $\left(\delta_{n}\right)$ and the shear displacement $\left(\delta_{s}\right) . \delta^{f}$ is the final displacement to complete failure, and $\delta^{o}$ is the displacement that (1) satisfied.

When $D$ remains zero, no slip occurs at the contact without separation in the normal direction. If $D$ is equal to unity, either full slip or complete separation occurs at the entire contact. The area within the stress-displacement curve corresponds to cohesive energy per unit area for friction and pull-off adhesion. In order to focus on friction, pull-off adhesion was excluded in this study.

It was identified from the literature that static friction between two solids increases with hold time, and timedependent frictional force $\left(Q^{\max }\right)$ is expressed with a simple $\log$ function [1]. The logarithmic increase of a frictional force is attributed to the deformation creep in plastic contacts:

$$
Q^{\max }(t)=Q_{0}^{\max }+\beta \cdot P \cdot \ln (t),
$$

where $t$ is hold time. $Q_{0}^{\max }$ is initial frictional force, $P$ is normal force, and $\beta$ is the constant. 
By dividing (3) with an actual contact area $(A)$, the maximum shear stress is given as

$$
\tau^{\max }(t)=\tau_{0}^{\max }+\beta \cdot \sigma_{c} \cdot \ln (t),
$$

where $\sigma_{c}$ is compressive stress.

Time-dependent cohesive energy, $G(t)$, can then be derived from a bilinear cohesive law (i.e., $G(t)=0.5 \cdot \tau^{\max }(t)$. $\left.\delta_{s}^{f}(t)\right)$. Consider

$$
G(t)=\frac{\left(\tau_{0}^{\max }+\beta \cdot \sigma_{c} \cdot \ln (t)\right) \cdot \delta_{s}^{f}(t)}{2},
$$

where $\delta_{s}^{f}(t)$ is total shear displacement leading to complete failure.

Meanwhile, the total shear displacement can be expressed with cohesive shear stiffness $\left(k_{s}\right)$ and the effective relative shear displacement to failure $\left(\Delta \delta_{s}^{f}\right)$ :

$$
\delta_{s}^{f}(t)=\frac{\tau^{\max }(t)}{k_{s}}+\Delta \delta_{s}^{f} .
$$

Measured results for silica $\left(\mathrm{SiO}_{2}\right)$ showed that cohesive stiffness and the effective relative shear displacement to failure $\left(\Delta \delta_{s}^{f}\right)$ were maintained as constant without regard to hold time.

Equation (5) can be rewritten as

$$
\begin{aligned}
G(t)= & \frac{\left(\tau_{o}^{\max }+\beta \cdot \sigma_{c} \cdot \ln (t)\right)^{2}}{2 k_{s}} \\
& +\frac{\left(\tau_{o}^{\max }+\beta \cdot \sigma_{c} \cdot \ln (t)\right)}{2} \Delta \delta_{s}^{f} .
\end{aligned}
$$

Figure 2 shows the frictional strength with respect to hold time. It is possible to express measured frictional strength with a simple log function as presented in the literature [4]. Figure 3 shows cohesive energy of $\mathrm{SiO}_{2}$ with respect to hold time. Markers show measured data of self-mated $\mathrm{SiO}_{2}$ specimens, and a smooth curve denotes a calculated one with (5). Good agreement was obtained between two values of cohesive energy data.

\section{Finite Element Simulation}

A three-dimensional model was generated by commercial finite element software (ABAQUS ${ }^{\circledR}$ ). The model consisted of a rounded tip (a radius of $15 \mathrm{~nm}$ ), a plate, and a cohesive layer between a tip and a plate. A cohesive layer was thin and circular. The diameter $(2 a)$ of a cohesive zone was determined by Hertz contact theory [11]:

$$
a=\left(\frac{3 P R}{4 E^{*}}\right)^{1 / 3}
$$

where $1 / E^{*}=\left(1-v_{1}^{2}\right) / E_{1}+\left(1-v_{2}^{2}\right) / E_{2}$. $P$ is normal force, $R$ is the radius of a rounded tip, $E_{1}$ and $\nu_{1}$ are elastic modulus and Poisson's ratio of a rounded tip, and $E_{2}$ and $\nu_{2}$ are elastic modulus and Poisson's ratio of a plate, respectively. Table 1
TABLE 1: Mechanical properties of silica $\left(\mathrm{SiO}_{2}\right)[10]$.

\begin{tabular}{lc}
\hline Elastic modulus $(\mathrm{GPa})$ & Poisson's ratio \\
\hline 68.9 & 0.17 \\
\hline
\end{tabular}

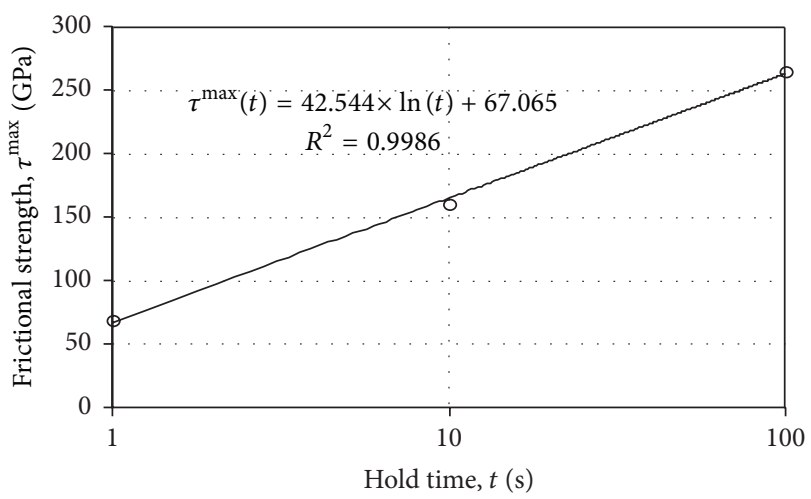

FIGURE 2: Frictional strength with respect to hold time on the semilog scale. Markers show measured data of self-mated $\mathrm{SiO}_{2}$ specimens at a normal force of $1 \mathrm{nN}$ and $60 \% \mathrm{RH}$ [4]. A smooth line denotes a fitted curve.

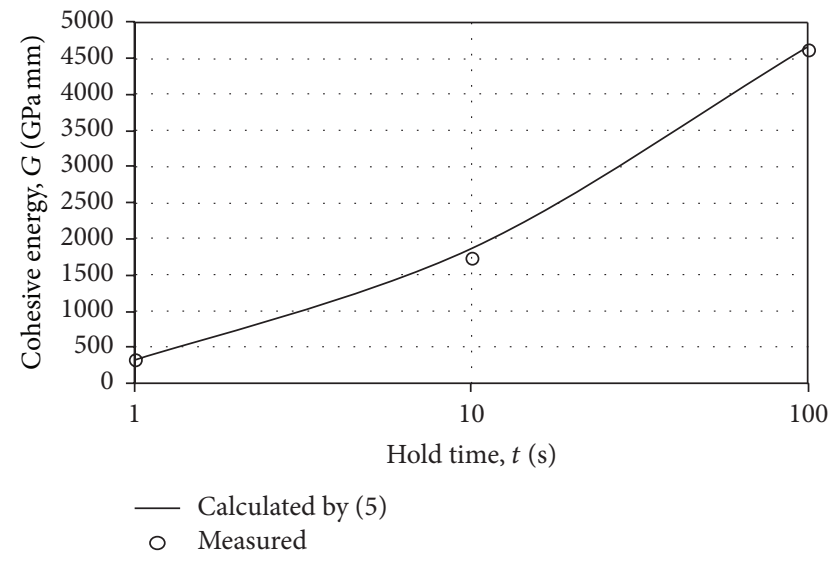

FIGURE 3: Cohesive energy of $\mathrm{SiO}_{2}$ for describing frictional aging. Markers show measured data of self-mated silica, and a smooth curve denotes a calculated one with (5).

shows elastic modulus and Poisson's ratio of silica used in this study.

An upper surface of a cohesive part was fixed to the contact surface of a tip in all directions, while the lower one of a cohesive part was fixed to a plate. A tetragonal element type was applied to the tip and the plate; the element size was $1 \mathrm{~nm}$. Parts of the tip and the plate contacted to a cohesive layer were meshed with an element of $0.1 \mathrm{~nm}$. All of the cohesive layer was meshed with a hexagonal element $0.05 \mathrm{~nm}$ long. The thickness of a cohesive layer $\left(h_{c}\right)$ was defined as $0.05 \mathrm{~nm}$. Table 2 shows the mechanical properties of a cohesive layer used in this study. The cohesive stiffness, the maximum nominal stress, and the effective displacement at failure were determined on the basis of experimental data [4]. The cohesive stiffness and the effective displacement at failure were assumed to be constant regardless of hold time. 
TABLE 2: Mechanical properties of a cohesive layer.

\begin{tabular}{lc}
\hline Properties & Value \\
\hline Shear stiffness, $k_{s},(\mathrm{GPa} \cdot \mathrm{nm})$ & 7.7 \\
Normal stiffness, $k_{n},(\mathrm{GPa} \cdot \mathrm{nm})$ & 7.7 \\
Max. nominal shear stress after a hold time of $1 \mathrm{sec} .(\mathrm{GPa})$ & 67.1 \\
Max. nominal tensile stress after a hold time of $1 \mathrm{sec} .(\mathrm{GPa})$ & 67.1 \\
The effective displacement at failure, $\Delta \delta^{f},(\mathrm{~nm})$ & 1.2 \\
\hline
\end{tabular}

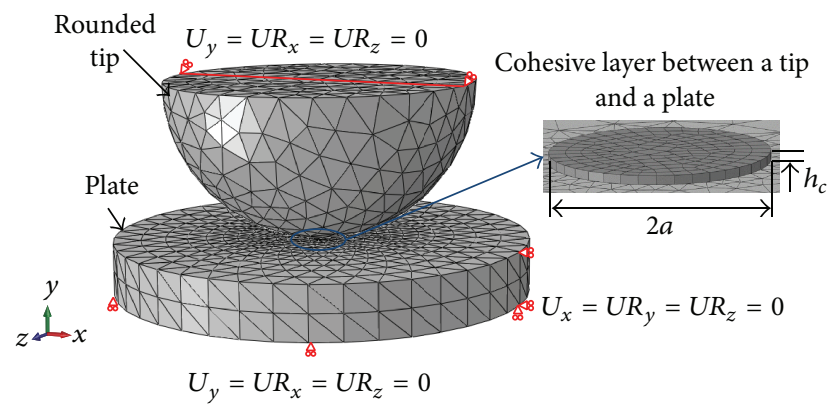

FIGURE 4: A finite element model with boundary conditions. $U$ and $U R$ denote displacement and rotation, respectively.

Initial sliding in a slide-hold-slide experiment was excluded in this simulation, since the initial sliding did not greatly affect frictional strength. Pressure of $1.41 \mathrm{MPa}$ (a normal force of $1 \mathrm{nN}$ ) was imposed to the upper surface of a tip. Then, the whole part of a tip was horizontally moved at a predescribed displacement, while the plate was fixed as shown in Figure 4.

\section{Results and Verification}

Implicit finite element modelling was employed to simulate frictional aging of self-mated silica. Cohesive energy presented in Figure 3 was used for describing the strengthening of bonding resulting from frictional aging. The maximum shear and normal stresses for a bilinear cohesive law were determined with time-dependent cohesive energy. After simulation, the maximum shear stress at the contact surface of a plate was recorded, along with the relative displacement between a tip and a plate. Frictional force was then calculated from the maximum shear stress.

Figure 5 shows the direct comparison between calculated and experimental frictional forces for the hold time of 1 second. Markers were measured data found in the literature [4] and a smooth curve was a calculated one. Excellent agreement was obtained between calculated and experimental contact stiffness values. In the plot, the maximum value was used for determining the coefficient of static friction. There was the difference of 11 percent between experimental and calculated values in the maximum frictional force. In addition, the decrease rate after the maximum frictional force was different.

Simulation for different hold time was employed. The effect of increased hold time in an experiment was reproduced with increase of cohesive energy presented in Figure 3.

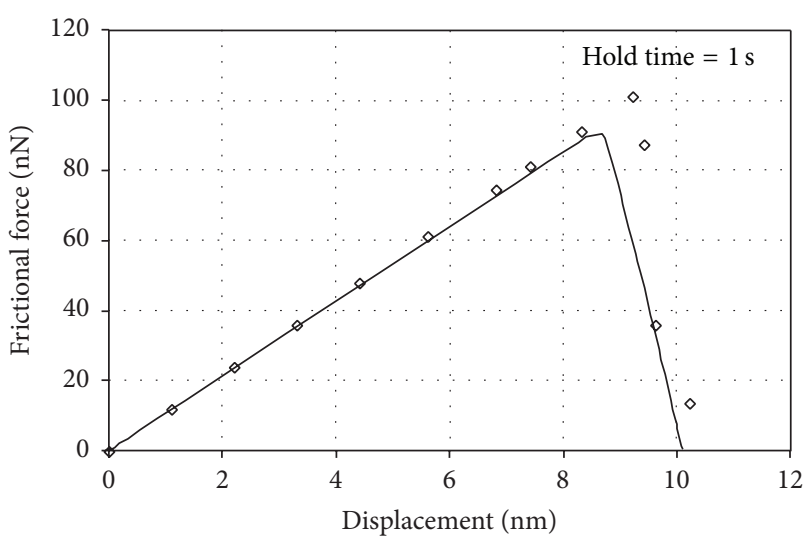

FIGURE 5: Direct comparison between measured and calculated frictional forces. Markers are measured data found in the literature [4] and a smooth curve is a calculated one.

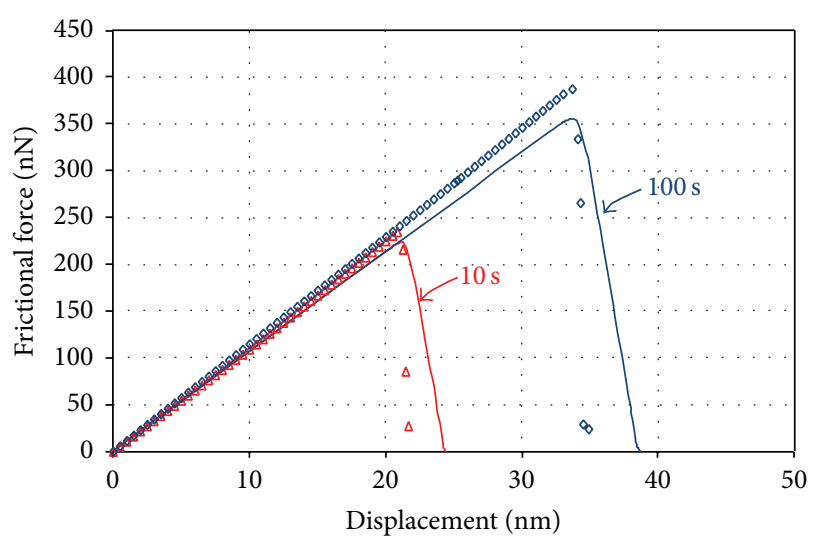

FIGURE 6: Comparison between calculated and experimental frictional forces after 10 and 100 seconds. Markers are measured data [4] and smooth curves are calculated ones.

Simulation was terminated when a cohesive zone was completely broken. Figure 6 shows calculated frictional forces after 10 and 100 seconds. A calculated frictional force for the hold time of 10 seconds was in excellent agreement with the experimental one (markers in Figure 6). However, the drop rate of the calculated force was different than that found in the experiment. The calculated maximum force for hold time of 100 seconds was 9 percent as low as the experimental one. Variance of chosen cohesive energy might be attributed to the difference in the maximum force. In this simulation, the effective displacement at failure for cohesive zone was constant without regard to hold time. This might be attributed to the difference between the drop rates of the force.

Figure 7 shows the dependence of cohesive element size in the maximum frictional force. In case the cohesive element size was $0.05 \mathrm{~nm}$, the difference between measured and calculated forces remained lower than 11 percent in the maximum frictional force. If the cohesive element size was greater than $0.1 \mathrm{~nm}$, the difference between measurement and calculation increased greatly (more than 17 percent). Figure 8 shows the dependence of cohesive thickness in the maximum 


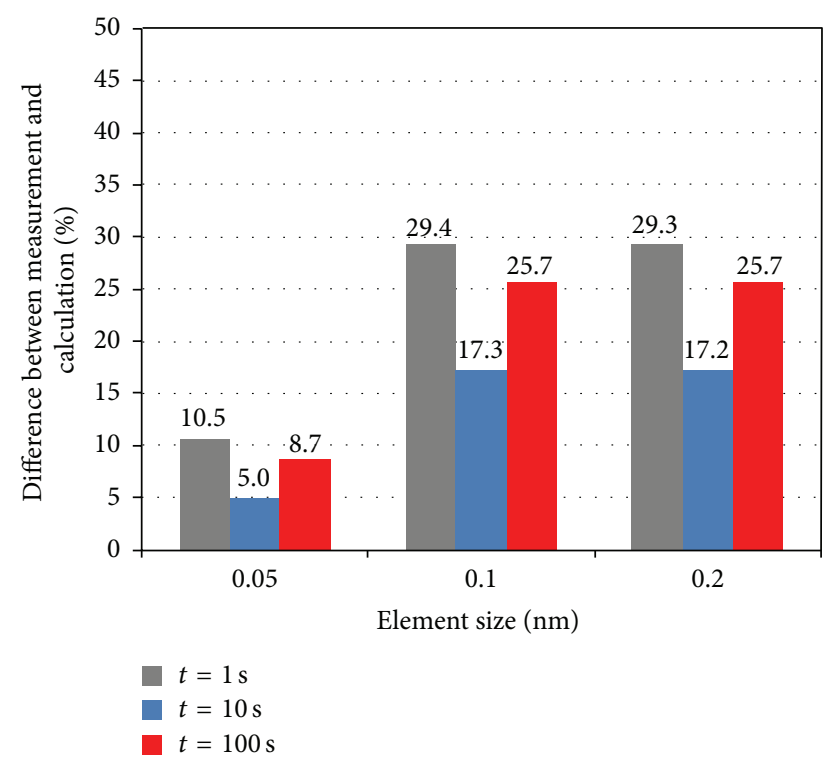

FIGURE 7: Dependence of cohesive element size in the maximum frictional force.

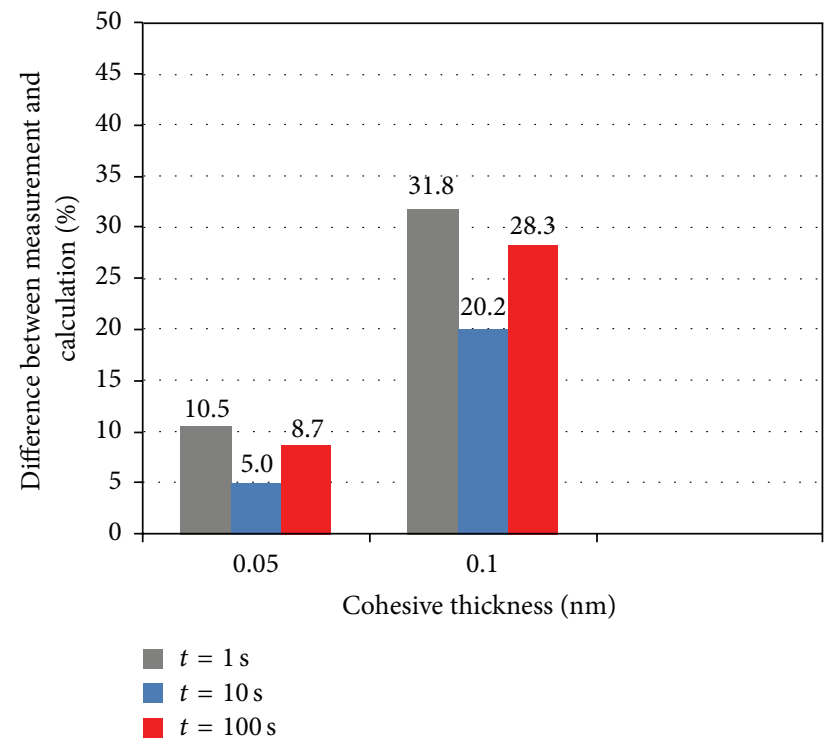

FIGURE 8: Dependence of cohesive thickness in the maximum frictional force. Cohesive element size was $0.05 \mathrm{~nm}$ in all cases.

frictional force; in case of a cohesive thickness of $0.1 \mathrm{~nm}$ and an element size of $0.05 \mathrm{~nm}$, the difference between measured and calculated forces was greater than 20 percent. Figures 7 and 8 provide adequate mesh size and cohesive thickness for obtaining accurate simulation result. That is, cohesive element size and cohesive thickness need to be lower than $0.05 \mathrm{~nm}$ to obtain the error of 10 percent in the maximum frictional force.

In this study, a bilinear cohesive zone law was applied to the modelling of frictional aging of self-mated silica. A proposed method allows simulating the strengthening of bonding resulting from frictional aging. The transition from partial slip to full slip was simulated with the softening of a cohesive zone law. The drop rates of the calculated frictional force after 10 and 100 seconds were somewhat different than experimental ones. Thus, further work needs to include an investigation into the difference between the drop rates. In addition, increase in contact area occurs during frictional aging. In this study, the contact area between silica materials was assumed to be constant regardless of hold time. Therefore, further modelling should include the increase in actual contact area with hold time.

\section{Conclusions}

The following conclusions were drawn:

(i) It was possible to simulate static friction between selfmated silica materials with a bilinear cohesive zone at the nanoscale.

(ii) Strengthening of interfacial bonding during frictional aging can be reproduced with the increased cohesive energy in a bilinear cohesive zone law.

(iii) An element size of $0.05 \mathrm{~nm}$ and a cohesive thickness of $0.05 \mathrm{~nm}$ were proposed to obtain simulation results with less than 10 percent in error.

Further work needs to simulate the transition from partial slip to full slip (kinetic friction). In addition, the increase of actual contact area during frictional aging needs to be included in a model.

\section{Nomenclature}

a: Actual contact radius

A: $\quad$ Actual contact area

$D$ : Damage variable

E: $\quad$ Modulus of elasticity

G: Time-dependent cohesive energy

$k_{s}: \quad$ Shear stiffness

$k_{n}: \quad$ Normal stiffness

$P: \quad$ Normal force

$Q^{\max }:$ Maximum frictional force

$Q_{0}^{\max }$ : Initial frictional force

$R: \quad$ Radius of a rounded tip

$t$ : Time

$\delta_{n}: \quad$ Normal displacement

$\delta_{s}: \quad$ Shear displacement

$\delta^{f}$ : Final displacement to complete failure

$\delta^{\text {max }}$ : Total displacement

$\delta^{o}: \quad$ Displacement at the maximum stress

$\delta_{s}^{o}$ : Shear displacement at the maximum shear stress

$\delta_{s}^{f}: \quad$ Final shear displacement to complete failure

$\delta_{n}^{o}: \quad$ Normal displacement at the maximum tensile stress

$\delta_{n}^{f}$ : Final normal displacement to complete failure

$v: \quad$ Poisson's ratio

$\sigma: \quad$ Tensile stress

$\sigma_{c}: \quad$ Compressive stress

$\sigma^{\max }:$ Maximum tensile stress

$\tau$ : $\quad$ Shear stress

$\tau^{\text {max }}$ : Maximum shear stress 
$\tau_{o}^{\max }:$ Initial shear stress

$\beta$ : Constant for time-dependent frictional force

$\Delta \delta_{s}^{f}$ : Effective relative shear displacement to failure.

\section{Conflict of Interests}

The authors declare that there is no conflict of interests regarding the publication of this paper.

\section{Acknowledgment}

This work was supported by 2016 Korea Aerospace University Faculty Research Grant.

\section{References}

[1] Y. Liu and I. Szlufarska, "Chemical origins of frictional aging," Physical Review Letters, vol. 109, no. 18, Article ID 186102, 2012.

[2] J. H. Dieterich, "Modeling of rock friction: 1. Experimental results and constitutive equations," Journal of Geophysical Research, vol. 84, no. 5, pp. 2161-2168, 1979.

[3] K. M. Frye and C. Marone, "Effect of humidity on granular friction at room temperature," Journal of Geophysical Research, vol. 107, no. B11, pp. ETG 11-1-ETG 11-13, 2002.

[4] Q. Li, T. E. Tullis, D. Goldsby, and R. W. Carpick, "Frictional ageing from interfacial bonding and the origins of rate and state friction," Nature, vol. 480, no. 7376, pp. 233-236, 2011.

[5] R. Capozza, I. Barel, and M. Urbakh, "Probing and tuning frictional aging at the nanoscale," Scientific Reports, vol. 3, article 1896, 2013.

[6] K. Kim, "Creep-rupture model of aluminium alloys: cohesive zone approach," Proceedings of the Institution of Mechanical Engineers, vol. 299, no. 8, pp. 1343-1347, 2015.

[7] K. Kim and M.-J. Yoon, "Fretting fatigue simulation for aluminium alloy using cohesive zone law approach," International Journal of Mechanical Sciences, vol. 85, no. 1, pp. 30-37, 2014.

[8] K. Kim, B. Forest, and J. Geringer, "Two-dimensional finite element simulation of fracture and fatigue behaviours of alumina microstructures for hip prosthesis," Proceedings of the Institution of Mechanical Engineers, Part H: Journal of Engineering in Medicine, vol. 225, no. 12, pp. 1158-1168, 2011.

[9] K. Kim, "Softening behaviour modelling of aluminium alloy 6082 using a non-linear cohesive zone law," Proceedings of the Institution of Mechanical Engineers, Part L: Journal of Materials: Design and Applications, vol. 229, no. 5, pp. 431-435, 2015.

[10] M. Zou and D. Yang, "Nanoindentation of silica nanoparticles attached to a silicon substrate," Tribology Letters, vol. 22, no. 2, pp. 189-196, 2006.

[11] A. Sackfield, D. A. Hills, and D. Nowell, Mechanics of Elastic Contacts, Butterworth-Heinemann, Oxford, UK, 1st edition, 1993. 

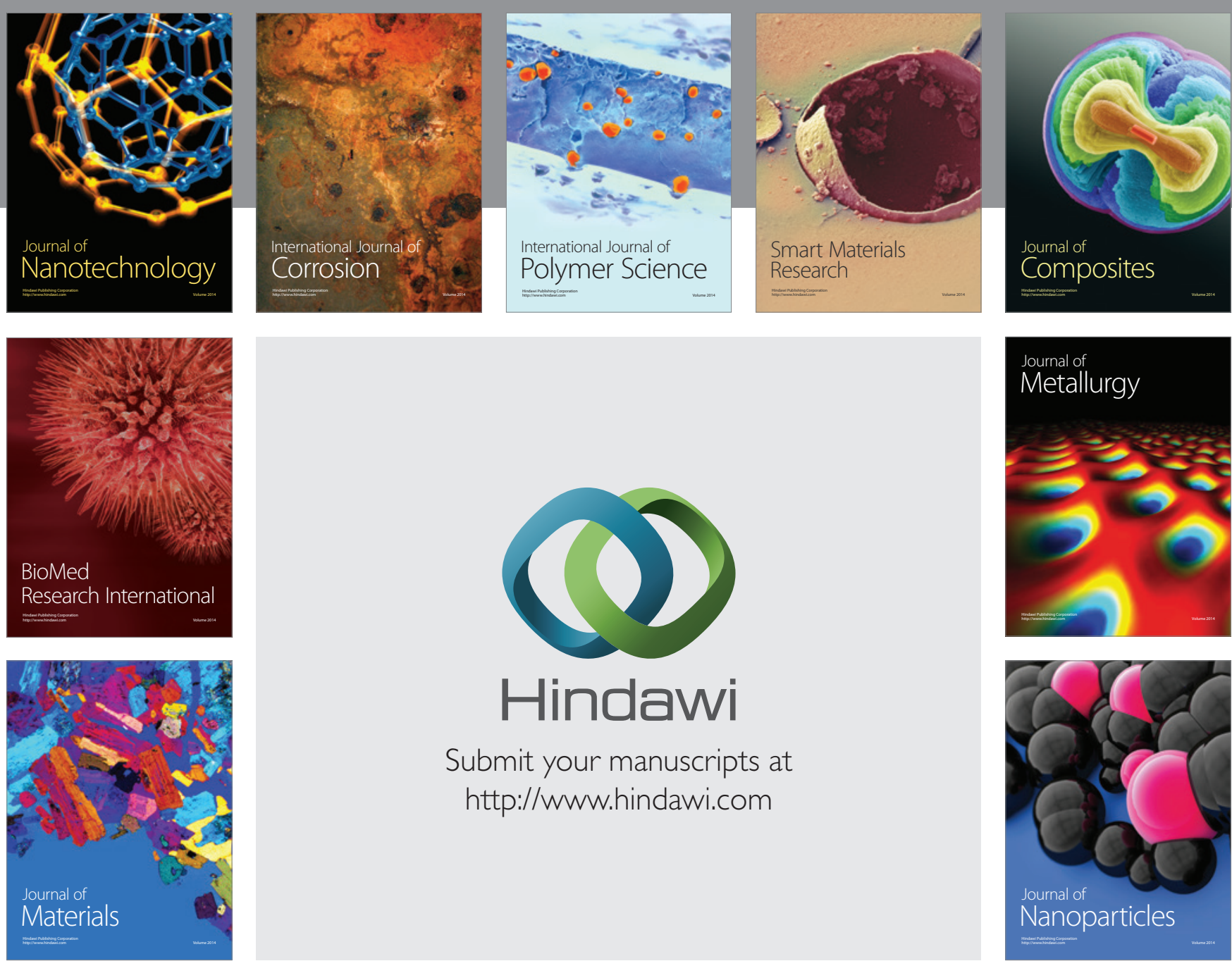

\section{Hindawi}

Submit your manuscripts at

http://www.hindawi.com

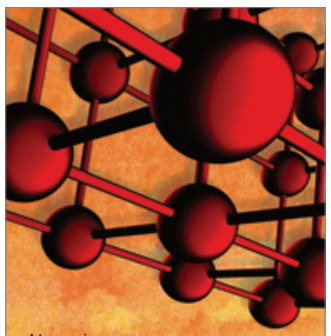

Materials Science and Engineering
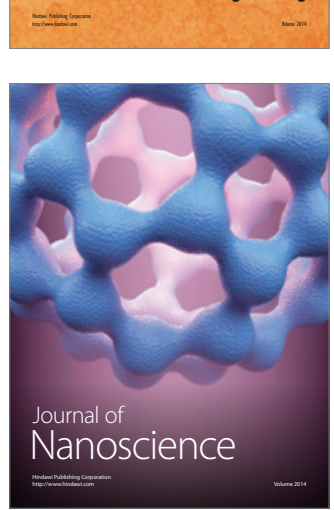
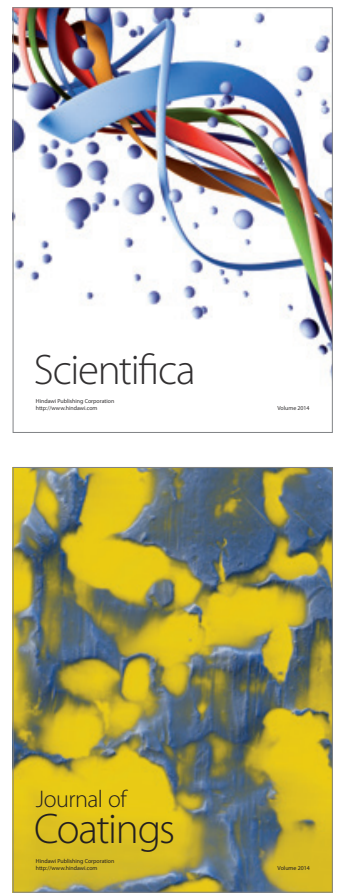
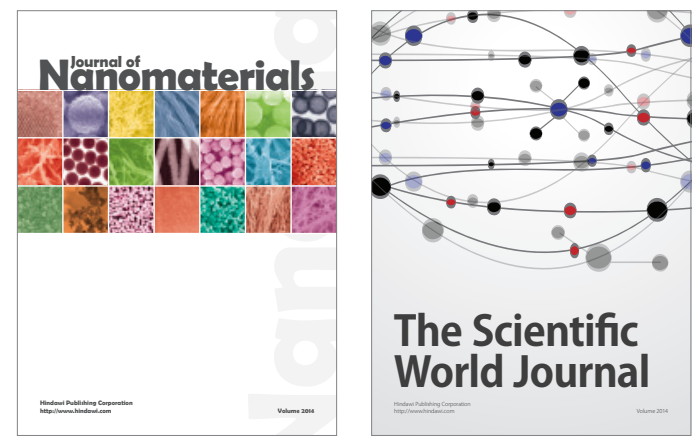

The Scientific World Journal
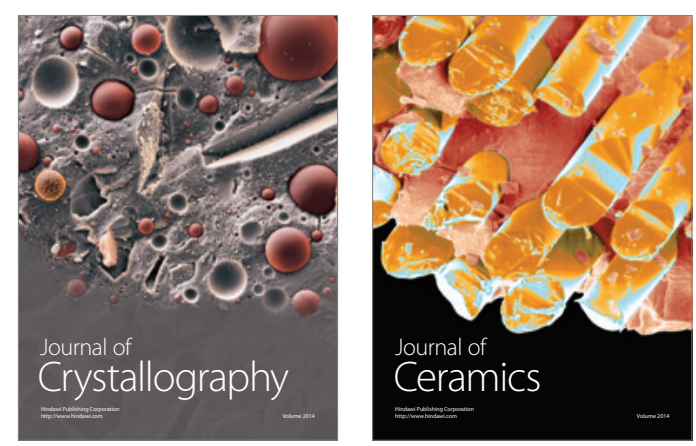
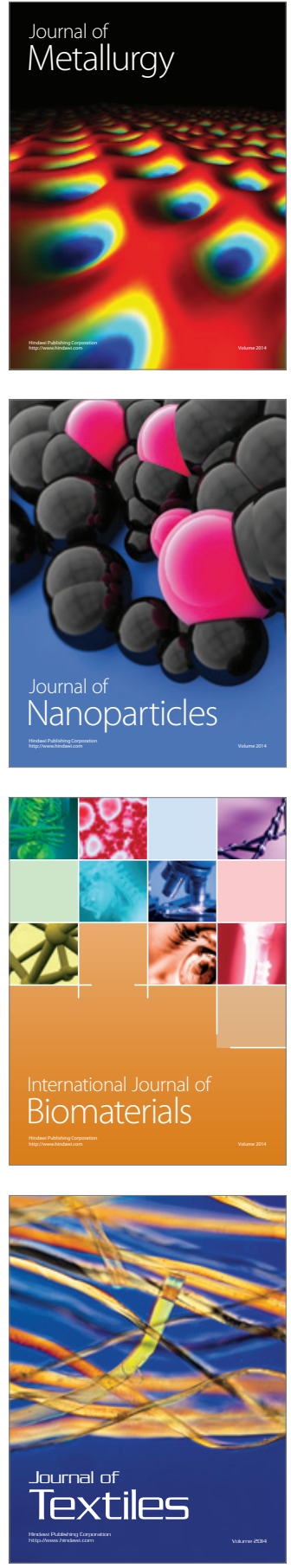